\title{
Prevalence of Signs and Symptoms of Dry Eye Disease 5 to 15 After Refractive Surgery
}

This article was published in the following Dove Press journal:

Clinical Ophthalmology

\author{
Bjørn Gjerdrum (1D ${ }^{1,2}$ \\ Kjell Gunnar Gundersen (D) ${ }^{2}$ \\ Per Olof Lundmark $\mathbb{D}^{\prime}$ \\ Rick Potvin (D) $^{3}$ \\ Bente Monica Aakre' \\ 'Department of Optometry, Radiography \\ and Lighting Design, University of South- \\ Eastern Norway, Kongsberg, Norway; \\ ${ }^{2}$ Ifocus Eye Clinic, Haugesund, Norway; \\ ${ }^{3}$ Science in Vision, Akron, NY, USA
}

Purpose: To compare the prevalence of dry eye disease (DED) as determined by signs and symptoms in patients with a history of laser vision correction (LVC) or implantable collamer lens (ICL) implantation 5-15 years ago with a matched control group with no history of refractive surgery.

Patient and Methods: This was a cross-sectional case-control study. The subject population included patients who had LVC or ICL 5 to 15 years ago. The control group was age matched. A test eye was randomly chosen. Subjects were required to have good ocular health. DED was evaluated using categorical cut-off criteria for tear film osmolarity (measured in both eyes), the subjective Ocular Surface Disease Index (OSDI), the dynamic Objective Scatter Index (OSI), non-invasive keratography tear break-up time (NIKBUT), meibography, and the Schirmer 1 test.

Results: The study included 257 subjects ( 94 LVC, 80 ICL, 83 control). The frequency of hyperosmolarity was significantly higher in the LVC group vs the control $(73 \%$ vs $50 \%, \mathrm{p}=$ 0.002), In contrast, the frequency of subjective symptoms tended to be lower in the LVC group than in the control group $(19 \%$ vs $31 \%$; $=0.06)$. These differences were not seen between the ICL and control group.

Conclusion: The results suggest that LVC may cause tear film instability as indicated by hyperosmolar tears up to 15 years after surgery, with few subjective symptoms of dry eye. This may have implications for IOL calculations for cataract or refractive lens exchange later in life.

Keywords: tear film, hyperosmolarity, OSDI, post LVC

\section{Introduction}

Cataract surgery and RLE are common surgical procedures where the natural crystalline lens of the eye is being replaced with an artificial intraocular lens (IOL). Calculations of IOL power depend on measurements (biometry) of (at a minimum) corneal curvature and axial length of the eye, but often include anterior chamber depth and lens thickness as well. In general, the accuracy of the procedure is high in patients without prior refractive surgery. However, for patients who have previously undergone laser treatment for myopia the precision is much lower, primarily due to 2 factors: inaccurate determination of the true total corneal refractive power and incorrect estimation of the effective lens position. ${ }^{1,2}$ Traditional optical biometers use reflections from the pre-corneal tear film to measure curvature as a part of the IOL power calculation. An uneven or unstable tear film due to dry eye may directly reduce the accuracy and repeatability of these measurements. ${ }^{3}$
Correspondence: Bjørn Gjerdrum Brønngata 36, Stavanger 4008, Norway Tel +474I5 II 935

Email bjorn@ifocus.no 
Dry eye disease (DED) is a common disease and clinical awareness has risen considerably around the world through the last three decades. ${ }^{4}$ The TFOS DEWS II (Tear Film and Ocular Surface Society International Dry Eye Workshop II) report has defined dry eye as

... a multifactorial disease of the ocular surface characterized by a loss of homeostasis of the tear film, and accompanied by ocular symptoms, in which tear film instability and hyperosmolarity, ocular surface inflammation and damage, and neurosensory abnormalities play etiological roles. $^{5}$

While this definition is helpful, there is a lack of standardized testing methods and criteria for categorizing dry eye. As such, reported prevalence ranges from $5 \%$ to $50 \%$ when based on signs and symptoms, and up to $75 \%$ based on signs only. ${ }^{5}$

Traditionally, classification has been based on consideration of the source - evaporative or aqueous deficient. The DEWS II revised classification indicates that these etiologies are overlapping. ${ }^{4}$ In a sense, all forms of DED are evaporative, because they are all associated with tear hyperosmolarity. ${ }^{6}$ The new DED definition emphasizes the role of homeostasis of the tear film, and diagnostic homeostasis marker tests are the minimum data set to be collected. $^{7}$ A recommended diagnostic test battery includes screening with a questionnaire, and homeostasis markers (non-invasive tear break-up time, osmolarity and staining). DED is diagnosed if the patient has symptoms and one of the homeostasis markers is positive, even without the full battery of recommended tests. ${ }^{7}$ Further testing of tear volume and lipids/meibomian glands is recommended for subtype classification before initiating appropriate treatment. ${ }^{7}$

Dry eye can be caused by different iatrogenic interventions including systemic or local drugs, contact lenses, eye surgery such as corneal refractive surgery and cataract surgery. ${ }^{8}$ Laser in situ keratomileusis (LASIK) surgery is among the most common operations performed worldwide, with more than 16 million procedures globally to 2015 and more than three million procedures in the US since $2015 .^{9,10}$ Dry eye is the most commonly reported problem following LASIK surgery. ${ }^{11,12}$ Corneal afferent nerve fibers are severed during flap creation and stromal ablation. The nerve damage interrupts the cornea to lacrimal gland reflex arc that impairs both basal and reflex tear secretion, reduces blink rate, and causes a disruption of the neurotrophic factors released from the corneal nerves. ${ }^{13}$ Tear osmolarity may increase as a result of decreased secretion of lacrimal gland protein, electrolyte and water secretion, and in addition a drop in the blink rate, with an increase in the evaporation of the tears. ${ }^{14}$ Increased tear osmolarity induces ocular surface inflammation by activating stress kinases which alter the ocular surface. ${ }^{14}$ Another mechanism associated with refractive surgery is LASIKinduced neurotrophic epitheliopathy (LINE), in which corneal staining is secondary to a reduction of blinking and a decreased release of neurotrophic factors. ${ }^{14,15}$ Other potential contributing factors include an inflammatory response to surgery and frequent use of eyedrops with preservatives, damage to the goblet cells by suction ring induced pressure, altered tear-film stability caused by changes in corneal curvature, medication-induced effects, and even discontinued wear of eyeglasses. ${ }^{14,16,17}$ For some patients, the sensations of dry eye could arise from spontaneous firing by the damaged or regenerating corneal peripheral nerves causing pain of neuropathic origin, or "phantom cornea". ${ }^{18}$ Almost all patients will have transient dry eye in the postoperative period but the estimates of prevalence vary widely with $40-59 \%$ at 1 month and $10-40 \%$ at 6 months. ${ }^{14,16,19,20}$ It is believed to resolve in most cases within the first postoperative year, but other studies have shown higher osmolarity 12 months after LASIK and that nerve regeneration may not be complete at 18 months. ${ }^{14,16,18,21}$ The majority of articles documenting dry eye after laser vision correction (LVC) surgery include only a limited time of observation after surgery. To the best of our knowledge, there are no studies evaluating dry eye as long as 5 years or more after refractive surgery.

The implantable collamer lens (ICL; STAAR Surgical, Monrovia, CA), a posterior chamber phakic IOL (pIOL), has a history of 30 years in refractive surgery around the world. $^{22}$ The procedure can be used to correct a higher range of ametropia than LVC. Some patients may be better candidates for ICL implantation due to pupil size, dry eyes, inadequate tissue volume for LASIK, abnormal topographic shape or personal preferences for a reversible procedure. ${ }^{23}$ While no studies specifically addressing dry eye after ICL implantation are evident in the literature, it is occasionally reported in general studies of the lens. In a study of 56 patients having ICL, two patients reported mild, and one reported moderate symptoms of dry eyes. ${ }^{23}$ Naj et al, in a meta-analysis of 7 studies (511 eyes) comparing iris fixated pIOL and ICL, reported 1 incident of clinical significant dry eye. ${ }^{24}$ Given the similarities of the ICL procedure to cataract or Refractive Lens Exchange 
(RLE) surgery, some of the same risk factors for dry eye should exist. Cataract surgery has been shown to independently transiently induce or exacerbate dry eye; studies have shown that dry eye symptoms increase after uncomplicated phacoemulsification but generally resolve after about 3 months. ${ }^{8}$ The signs associated with post-cataract dry eye include decrease in tear break up time, increased ocular surface staining and changes in tear volume. The presumed pathophysiological mechanisms underlying cataract surgery induced dry eye include use of topical anesthetics, exposure desiccation, possible light toxicity from the operating microscope, nerve transection, elevation of inflammatory factors, goblet cell loss, and meibomian gland dysfunction (MGD). ${ }^{8}$ The surgical trauma may also affect corneal sensitivity, increase inflammation and contribute to tear film instability. ${ }^{8}$

Since data were available for the ICL patients and limited information exists in the literature on the frequency of DED in this group, we chose to include these patients in our study. ICL implantations are not associated with dry eyes or reduced precision in IOL calculations so the ICL group serves as an extra control group. The aim of this study was to compare the prevalence of DED as determined by different signs and symptoms in patients undergoing LVC or ICL 5 to 15 years ago to a similar population with no history of refractive surgery, as unstable tear film may be a confounding source of error in calculating IOL-power in post-LVC patients. Long-term observation data can add to our understanding of these sources of error in IOL calculation for post-LVC patients in particular, to determine if it needs to be given extra consideration in this population.

\section{Patients and Methods}

The study was a cross-sectional case-control study involving data from the Ifocus private eye clinic in Haugesund, Norway. Participants were recruited from patients who had undergone LVC (LASIK or Femto-LASIK) or ICL 5-15 years ago. All surgeries were performed by the same surgeon. LASIK surgeries were performed with Amadeus II microkeratome with superior hinge and 130-micron flap thickness. Femto-LASIK (1 subject) were performed with Wavelight FS 200 with superior hinge and 110-micron flap thickness. ICL surgery was performed with a temporal $2,75 \mathrm{~mm}$ main incision and two side ports at 60 degrees from the main incision. The anterior chamber was filled with viscoelastic, and the ICL (STAAR Surgical Company, Lake Forest, CA, USA) was implanted into the anterior chamber. The haptics were positioned behind the iris into the sulcus. Toric lenses were rotated to the planned axis. Surgical iridectomy was performed near 12 o'clock position. Viscoelastic was removed and pupil contracted using Miochol-E (Bausch \&Lomb Bridgewater, NJ 08807 USA)

Patients from a population who were pre-examined or screened and found eligible for refractive surgery but who had elected not to proceed were age matched and recruited as controls. Eligible participants were identified from clinical patient records, randomly selected and consecutively recruited (by telephone, e-mail, or text message). Recruitment and data collection were performed from March 2018 to January 2019. The study followed the tenets of the Declaration of Helsinki and was approved by the Regional Committee for Medical and Health Research Ethics in Norway (Ref no 2018/75). A written informed consent was obtained.

Inclusion criteria were age over 20 years at the time of original surgery, bilaterally good ocular health, with no pathology or systemic disease involving the corneal surface, and corrected visual acuity $\geq 0.1 \log$ MAR at the time of recruitment. Exclusion criteria were manifest corneal scarring, lid deformities, any acute or chronic disease or illness that would confound the results of the study, pregnancy or lactation, recent intra- or extra-ocular surgery, ICL patients who have had a subsequent corneal refractive surgery (laser touch-up), previous radial keratotomy, or other corneal surgery besides LASIK (e.g. photorefractive keratectomy (PRK), Laser-assisted subepithelial keratectomy (LASEK), transplant, lamellar keratoplasty). Patients were instructed to not wear contact lenses on the examination day and/or not to use any eyedrops for at least $2 \mathrm{~h}$ before the examination.

One eye was randomly selected as the test eye. Uncorrected distance visual acuity (UDVA), refraction and corrected distance visual acuity (CDVA) were tested after osmolarity and the other tests in the order described below. A timespan of at least 5 mins was given between the HD-analyzer and the Keratograph, to allow for stabilization of the tear film. If some measurements were not possible to obtain because of eyemovements, blinking or other reasons, these patients were rescheduled (if possible), and a complete new set of measurements was taken. Otherwise, the test was recorded as $\mathrm{n} / \mathrm{a}$. Visual acuity was recorded on a Snellen chart and converted to $\log$ MAR. All testing was done by one clinician (B.G.). 


\section{Tear Film Osmolarity}

Tear film osmolarity was measured with the Tearlab Osmolarity System (Tearlab Corp., Escondido, California, USA) Tear film osmolarity was selected as the primary outcome measure for the study, as it is documented to have an effect on repeatability of keratometry. ${ }^{3}$ Osmolarity was always the first test on all patients, and both eyes were measured as recommended by the manufacturer and because commonly used criteria for DED involve the osmolarity in both eyes. Testing was performed as described by the manufacturer. ${ }^{25}$ It is suggested that a cut-off of $316 \mathrm{mOsm} /$ $\mathrm{L}$ is best for diagnosing moderate to severe DED. Furthermore, a between-eye difference $\geq 8 \mathrm{mOsm} / \mathrm{L}$ is a sign of loss of tear film homeostasis. ${ }^{7}$ As such, the cutoff criteria for categorizing hyperosmolarity in this study were the worse eye having an osmolarity of $\geq 316 \mathrm{mOsm} / \mathrm{L}$ or a between-eye difference $\geq 8 \mathrm{mOsm} / \mathrm{L}$.

\section{Dynamic Ocular Scatter Index (OSI)}

The optical quality of the tear film was assessed with the HD Analyzer quality analysis system (OQAS) (HD analyzer, Visiometrics S.L., Terrassa, Spain). Details of the system and testing procedure are described elsewhere. ${ }^{26}$ The dynamic Ocular Scatter Index (OSI) is recorded for a total of $20 \mathrm{~s}$. For each patient measurement, the device calculates the mean OSI, the standard deviation (OSI St.d) and the difference (OSI difference) between maximum (OSI max) and minimum OSI (OSI min). The Vision Break-Up Time (VBUT) is the time in seconds (maximum $10 \mathrm{~s}$ ) before the subject's OSI increases one unit from the minimum observed value. The changes in OSI (OSI std, OSI difference, and VBUT) are a result of tear film dynamics as other opacities in the cornea, lens or vitreous body do not change during the interblink interval. ${ }^{27,28}$ The summary statistics for the OSI mean, OSI Standard Deviation, OSI Difference and VBUT for all patients were reported. The cut-off criteria for categorizing DED were a $\operatorname{VBUT}<10 \mathrm{~s}$.

\section{Subjective OSDI Questionnaire}

The OSDI questionnaire is a validated and widely used questionnaire for clinical trials related to the eye. ${ }^{7,29}$ Using a total of 12 questions with a score from 0 to 4 , the OSDI score is obtained by multiplying the sum by 25 and dividing by the number of questions answered. This yields a score from 0 to 100 , with higher scores representing greater disability. ${ }^{29}$ For this study, only the total score was recorded. The cut-off criteria for categorizing DED were an OSDI score $\geq 13$. $^{7}$

\section{Non-Invasive Keratograph Break Up Time (NIKBUT)}

NIKBUT was assessed using the Oculus Keratograph 5M (Oculus, Wetzlar, Germany). Placido rings are reflected on the corneal surface. The system detects distortion in the reflected mires which is recorded as a break in the tear film. Details of the system and testing procedure are described in the instruction manual and user guide. ${ }^{30,31}$ The system detects the 1st break-up time and average break-up time (NIKBUT average) but only the latter was reported in this study. Based on studies of fluorescein break-up time (FBUT) and comparison between FBUT and NIKBUT, our cut-off criteria for categorizing DED were NIKBUT average $\leq 10$ seconds. ${ }^{7,32-35}$

\section{Meibography}

Meibography was assessed using the meiboscan function of the Oculus Keratograph 5M (Oculus, Wetzlar, Germany). Meibography allows observation of the silhouette of the meibomian gland morphological structure. ${ }^{7}$ Details of the system, testing procedure and grading are described in the instruction manual and user guide. ${ }^{30,31}$ Results were recorded on a 0-3 (0.5 step) continuous scale: Grade 0 (no loss of meibomian glands), Grade 1 (0-1/3 loss), Grade 2 (1/3-2/3 loss) and grade 3 (loss $>2$ / $3)$. A study by Arita et al considered a summed meiboscore of upper and lower eyelid $\geq 3$ as abnormal. ${ }^{36}$ For this study assessment of the lower eye lid was considered sufficient. $^{37}$ Based on this our criteria for categorizing DED was a lower eyelid meiboscore of $\geq 1.5$.

\section{Schirmer I}

The Schirmer test was performed without anaesthesia (Schirmer 1) using a Schirmer paper strip (HUB Pharmaceuticals, Rancho Cucamonga, CA). It is a standardized test, providing an estimation of stimulated reflex tear flow. ${ }^{7}$ Details of the testing procedure are described elsewhere. ${ }^{7}$ The cut-off criteria for categorizing DED was $\leq 10 \mathrm{~mm}$ after $5 \mathrm{mins}$, a threshold that is commonly accepted in clinical trials. ${ }^{38}$

\section{Analysis}

Data were recorded on an Excel spreadsheet (Microsoft Corp., Redmond, WA, USA). The data file from the HD 
Analyzer and the exported NIKBUT data from the Keratograph were transferred to the data in the spreadsheet, and cross checked. Descriptive statistics included the minimum, maximum, mean, standard deviation and the interquartile range (IQR). Statistical analysis was performed using $t$-test, ANOVA or nonparametric tests as appropriate and Pearson $\chi^{2}$ test was used for comparing frequencies. Missing data were not included in the analysis. A p-value 0.05 (two-sided) was considered statistically significant. Statistical analyses were performed using the RStudio data-analysis software (version 1.2.1335) RStudio Inc (Boston, MA, USA) and R Commander (version 2.60) (R Core Team, Vienna, Austria).

\section{Post Hoc Analysis}

Correlations between osmolarity and other factors known to affect dry eye (like age, sex, preoperative refraction, time of day, and season), and between minimum OSI and dynamic OSI were tested with Pearson's correlation coefficient of determination or Spearman's rank correlation for nonparametric variables.

\section{Results}

Subject demographics and refractive error are shown in Table 1. A total of 893 patients were examined for eligibility, 661 were found eligible, but 242 could not be reached or lived too far away. Of the remaining 419 patients, 96(74\%), 80(85\%) and 85(67\%) were recruited in the LVC, ICL and control group, respectively. One patient was excluded because of possible systemic disease, two were excluded because of LVC surgery less than five years ago, and one was excluded because of lactation. A total of 257 patients were included in the study: 94 (45 females, 49 males) in the LVC group, 80 (57 females, 23 males) in the ICL group and 83 (41 females, 42 males)

Table I Demographics, Pre- and Postoperative Refraction and Visual Acuity

\begin{tabular}{|c|c|c|c|c|c|}
\hline & & LVC, $n=94$ & ICL, $n=80$ & CTRL, $n=83$ & p \\
\hline & & $\begin{array}{l}\text { Mean } \pm \text { SD } \\
\text { (Range) }\end{array}$ & $\begin{array}{l}\text { Mean } \pm \text { SD } \\
\text { (Range) }\end{array}$ & $\begin{array}{l}\text { Mean } \pm \text { SD } \\
\text { (Range) }\end{array}$ & \\
\hline Sex: $f \%$ & & $47.9 \%$ & $71.2 \%$ & $49,4 \%$ & $0.003^{a *}$ \\
\hline Age, year & & $\begin{array}{l}41.3 \pm 6.3 \\
(29 \text { to } 57)\end{array}$ & $\begin{array}{l}40.8 \pm 8.8 \\
(25 \text { to } 64)\end{array}$ & $\begin{array}{l}4 I .2 \pm 8.1 \\
(23 \text { to } 56)\end{array}$ & $0.905^{\mathrm{b}}$ \\
\hline Years sin & & $\begin{array}{l}7.7 \pm 1.3 \\
(5.2 \text { to } 12.8)\end{array}$ & $\begin{array}{l}10.2 \pm 3.1 \\
(5.0 \text { to } 14.7)\end{array}$ & & $<0.001^{\mathrm{c}}$ \\
\hline Pre-Tx & MRSE, DS & $\begin{array}{l}-2.76 \pm 1.75 \\
(-8.00 \text { to }+2.37)\end{array}$ & $\begin{array}{l}-6.10 \pm 5.16 \\
(-17.12 \text { to }+8.00)\end{array}$ & $\begin{array}{l}-1.38 \pm 3.45 \\
(-9.37 \text { to }+6.87)\end{array}$ & $<0.00 I^{d *}$ \\
\hline & CYL, DC & $\begin{array}{l}-0.94 \pm 0.88 \\
(-3.50 \text { to } 0)\end{array}$ & $\begin{array}{l}-1.40 \pm 1.43 \\
(-8.75 \text { to } 0)\end{array}$ & $\begin{array}{l}-0.87 \pm 1.20 \\
(-7.00-0)\end{array}$ & $0.001^{e *}$ \\
\hline & BCVA, (logMAR) & $\begin{array}{l}-0.05 \pm 0.04 \\
(-0.18 \text { to } 0.05)\end{array}$ & $\begin{array}{l}0.00 \pm 0.07 \\
(-0.18 \text { to } 0.3)\end{array}$ & $\begin{array}{l}-0.06 \pm 0.07 \\
(-0.18 \text { to } 0.10)\end{array}$ & $<0.001^{\mathrm{e} *}$ \\
\hline Post-Tx & MRSE & $\begin{array}{l}-0.07 \pm 0.38 \\
(-2.37 \text { to }+0.75)\end{array}$ & $\begin{array}{l}-0.19+0.59 \\
(-2.25 \text { to }+1.50)\end{array}$ & & $0.017^{\mathrm{f} *}$ \\
\hline & CYL & $\begin{array}{l}-0.19 \pm 0.25 \\
(-1.0 \text { to } 0)\end{array}$ & $\begin{array}{l}-0.38 \pm 0.38 \\
(-1.50 \text { to } 0)\end{array}$ & & $<0.00 \mathrm{I}^{\mathrm{f} *}$ \\
\hline & UCVA (logMAR) & $\begin{array}{l}-0.03 \pm 0.12 \\
(-0.18 \text { to } 0.70)\end{array}$ & $\begin{array}{l}0.06 \pm 0.16 \\
(-0.18 \text { to } 0.7)\end{array}$ & & $<0.00 \mathrm{I}^{\mathrm{f} *}$ \\
\hline & BCVA (logMAR) & $\begin{array}{l}-0.07 \pm 0.05 \\
(-0.18 \text { to } 0.02)\end{array}$ & $\begin{array}{l}-0.03 \pm 0.06 \\
(-0.18 \text { to } 0.10)\end{array}$ & & $<0.00 I^{f} *$ \\
\hline
\end{tabular}

Notes: a Pearson's $\chi^{2}$ test ICL difference from CTRL, ${ }^{b}$ Anova (unequal variance), 'Wilcoxon rank-sum test between ICL/LVC, ${ }^{d} \mathrm{Kruskal}-\mathrm{Wallis}$ rank-sum test, ${ }^{\mathrm{e} W i l c o x o n}$ rank-sum test difference between ICL and CTRL, 'Wilcoxon rank-sum test, *Statistically significant.

Abbreviations: LVC, Laser Vision Correction; ICL, Implantable Collamer Lens; CTRL, Control group; MRSE, mean spherical equivalent refraction; CYL, refractive cylinder; BCVA, best-corrected visual acuity (logMAR); UCVA, uncorrected visual acuity (logMAR); Pre-Tx, historic data before surgery; Post-Tx, post-treatment data (study examination). 
in the control group. The ICL group had significantly more females vs the control group and significantly longer time since surgery vs the LVC group. There were significant differences in preoperative refraction between groups.

Table 2 summarizes the mean values of the various testing results. None of the tests showed significant differences in mean values except for the OSI measures. The OSI measures were significantly higher in the ICL group compared to the control group. However, the minimum OSI was significantly correlated to OSI Standard Deviation, OSI Difference, and VBUT (Spearman's Rho: $0.43,0.45$ and $-0,33$, respectively, $\mathrm{p}<0.01$ ) for all subjects.

When results were categorized according to the cut-off criteria described in the methods (Table 3), the frequency of hyperosmolarity was significantly higher in the LVC group vs the control group ( $73 \%$ vs $50 \%$ ), but not significantly different between the ICL and control group (Figure 1). The frequency of VBUT $\leq 10 \mathrm{~s}$ was significantly higher in the ICL group vs the control group (33\% vs $17 \%$ ). No other single objective tests or combination of criteria showed any significant difference between LVC or ICL and the control group. The frequency of OSDI $\geq 13$ tended to be lower in the LVC group relative to the control (19\% vs 31\%); this was not statistically significant $(p=0.06)$. The frequency of OSDI $\geq 13$ in the ICL group was the same as the control $(31 \%)$.

We could not establish any significant correlation between osmolarity and any of the other single DED tests. However, the frequency of hyperosmolarity was significantly higher in patients with two or more other indicators of DED (66\% vs $52 \% \mathrm{mOsm} / \mathrm{L}, \mathrm{p}=0.03)$.

There was no significant correlation between osmolarity and pre-operative mean spherical equivalent refraction (pre-MRSE) for the LVC group alone. Stepwise multivariate analysis including all patients tended towards a positive correlation between osmolarity and age and pre-MRSE (Pearson's $\mathrm{R}^{2}=0.08, \mathrm{p}<0.01$ and 0.03 , respectively). While significant, these correlations are weak. An example of this is shown in Figure 2; it can be seen that several outliers are influencing the fit. The single eye osmolarity cut-off value of $316 \mathrm{mOsm} / \mathrm{L}$ is shown for reference.

\section{Discussion}

The main objective was to compare the prevalence of DED as determined by different signs and symptoms in patients with previous refractive surgery to a control group, because this may affect keratometry measurement and therefore IOL calculation at the time of cataract surgery. Epitropoulos et al compared repeatability of keratometry in a hyperosmolar and a normal group. In the hyperosmolar group, $8 \%$ had a difference of more than $0.50 \mathrm{D}$, and $5 \%$ had a difference of more than $1 \mathrm{D}$ while all subjects had less than $0.5 \mathrm{D}$ in the normal group. ${ }^{3}$ For a patient with previous myopic LVC, a difference in keratometry of $1 \mathrm{D}$ could give approximately $0.8 \mathrm{D}$ to $1.2 \mathrm{D}$ difference in refractive outcome when using post-LVC IOL calculation formulas. ${ }^{39}$ The contribution of errors from tear-film instability could be relatively small when compared to sources of error like keratometric index error and incorrect estimate of the effective lens position. However, these errors are attempted solved in the post-LVC IOL formulas. While average prediction error for several Post-LVC formulas are within \pm 0.5 , it could range from $+1 \mathrm{D}$ to $-2 \mathrm{D} .^{40,41}$ Arguably, in the cases with highest prediction errors, several factors probably contribute, like diameter error, actual IOL position, and erroneous keratometric measurement due to unstable tear film may be another. Therefore, it is interesting to know if previous LVC patients have higher risk of unstable tear film than patients without prior refractive surgery.

Although not shown in mean osmolarity of the test eye, when using cut-off values as described, we found that the prevalence hyperosmolarity was significantly higher in the LVC group vs the control group and the ICL group. This is likely a consequence of the fact that both intra- and inter-eye variability of osmolarity is a hallmark of DED. ${ }^{42}$ The prevalence of DED in both the LVC group and the control group was relatively high compared to some other studies. One study reported osmolarity greater than $308 \mathrm{mOsm} / \mathrm{L}$ in $30 \%$ at 12 months after LASIK. ${ }^{21}$

De Paiva et al found that dry eye was associated with preoperative myopia and ablation depth at 6 months after surgery, possibly because of nerves needing to regenerate a longer distance in the case of deeper ablation depth. ${ }^{20} \mathrm{We}$ did not find correlation between pre-MRSE and Osmolarity in the LVC group. This difference may be explained in that our subjects had 5 years or more since surgery and differences in regeneration due to ablation depth have been leveled out. A meta-analysis by Feng et al found significantly higher tear-BUT, less loss of sensation and less corneal staining in patients with horizontal hinge flap compared to superior hinge flaps, but all our patient had nasal hinge except for one Femto-LASIK patient. $^{16}$

A meta-analysis in the DEWS II epidemiology report found prevalence of DED in the general population varying from $14 \%$ to $39 \%$ based on symptoms, and $16 \%$ to $86 \%$ based 


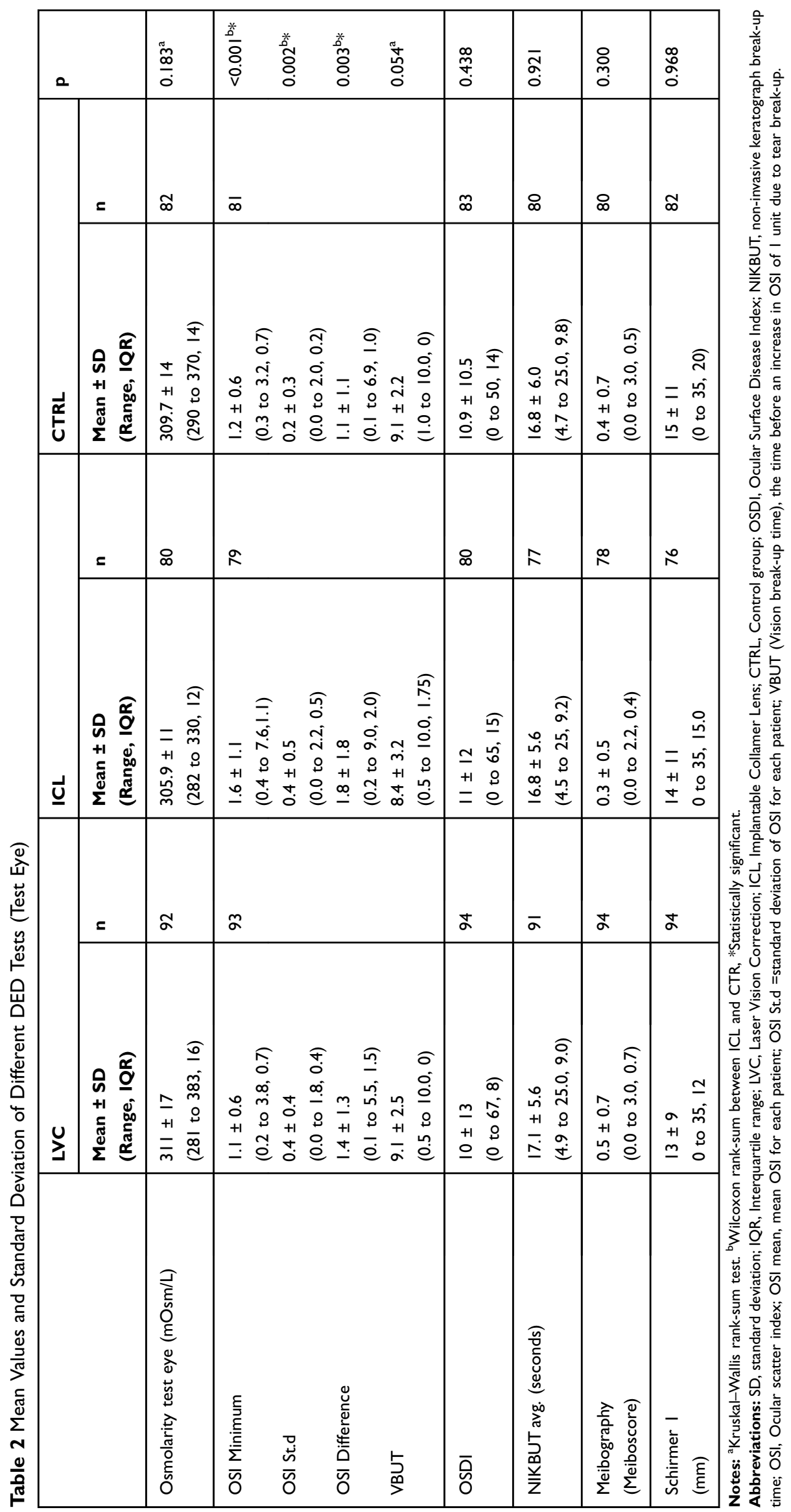


Table 3 Prevalence of DED as Determined by Signs and Symptoms

\begin{tabular}{|c|c|c|c|c|c|c|c|c|}
\hline \multirow{2}{*}{$\begin{array}{l}\text { Treatment group } \\
\text { Test variable (Cut-off values) }\end{array}$} & \multicolumn{3}{|l|}{ LVC } & \multicolumn{3}{|l|}{ ICL } & \multicolumn{2}{|c|}{ CTRL } \\
\hline & $\%$ & $\mathbf{n}$ & $\mathbf{p}^{\mathbf{a}}$ & $\%$ & $\mathbf{n}$ & $\mathbf{p}^{\mathbf{a}}$ & $\%$ & $\mathbf{n}$ \\
\hline $\begin{array}{l}\text { Osmolarity } \\
\text { ( } \geq 316 \text { either eye or } \geq 8 \text { inter-eye diff.) }\end{array}$ & $73.3 \%$ & 90 & $0.002 *$ & $46.2 \%$ & 80 & 0.63 & $50 \%$ & 82 \\
\hline VBUT ( $\leq 10$ seconds) & $24.7 \%$ & 93 & 0.23 & $32,9 \%$ & 79 & $0.02 *$ & $17,3 \%$ & 81 \\
\hline OSDI $(\geq 13)$ & $19.1 \%$ & 94 & 0.06 & $31.2 \%$ & 80 & .99 & $31.3 \%$ & 83 \\
\hline $\begin{array}{l}\text { NIKBUT avg } \\
\text { ( } \leq 10 \text { seconds) }\end{array}$ & $12.1 \%$ & 91 & 0.32 & $15.6 \%$ & 77 & 0.75 & $17.5 \%$ & 80 \\
\hline $\begin{array}{l}\text { Meibography } \\
\text { (meiboscore } \geq 1.5 \text { ) }\end{array}$ & $10.6 \%$ & 94 & 0.68 & $5.1 \%$ & 78 & 0.37 & $8.8 \%$ & 80 \\
\hline $\begin{array}{l}\text { Schirmer } \\
\text { (mm wetting } \leq 10 \mathrm{~mm})\end{array}$ & $51.1 \%$ & 94 & 0.76 & $48.7 \%$ & 76 & 0.99 & $48.8 \%$ & 82 \\
\hline OSDI and one other indicator & $18.1 \%$ & 94 & 0.55 & $27,5 \%$ & 80 & 0.39 & $21.7 \%$ & 83 \\
\hline
\end{tabular}

Notes: ${ }^{\text {Pearson's }} \chi^{2}$ : difference from control. *Statistically significant.

Abbreviations: LVC, Laser Vision Correction; ICL, Implantable Collamer Lens; CTRL, Control group; OSDI, Ocular Surface Disease Index; NIKBUT avg, average noninvasive keratograph break-up time; VBUT, HD analyzer Vision break up time.

on signs. $^{43}$ Gupta et al found abnormal osmolarity $(>307 \mathrm{mOsm} / \mathrm{L}$ in either eye or an inter-eye difference $>7 \mathrm{mOsm} / \mathrm{L}$ ) in $57 \%$ of 120 patients (including 25 patients with previous refractive surgery) presenting for cataract surgery. $^{44}$

The relative high prevalence of DED in all groups could possibly be related to the fact that many patients who have problems with contact lenses due to dry eyes consider refractive surgery as a solution, and up to $73 \%$ of
LVC patients have been reported to seek surgery because of difficulties with contact lens wear. ${ }^{14,45}$ There are several risk factors for developing dry eye after LASIK, with preexisting dry eye being the most significant. ${ }^{14,46}$ Konomi et al suggested that lower preoperative tear volume may increase the risk of chronic dry eye. ${ }^{47}$ In addition, age is a risk factor for DED and the LVC and ICL groups in this study were on average 8 and 10 years older, respectively, than at time of their surgery. ${ }^{6}$

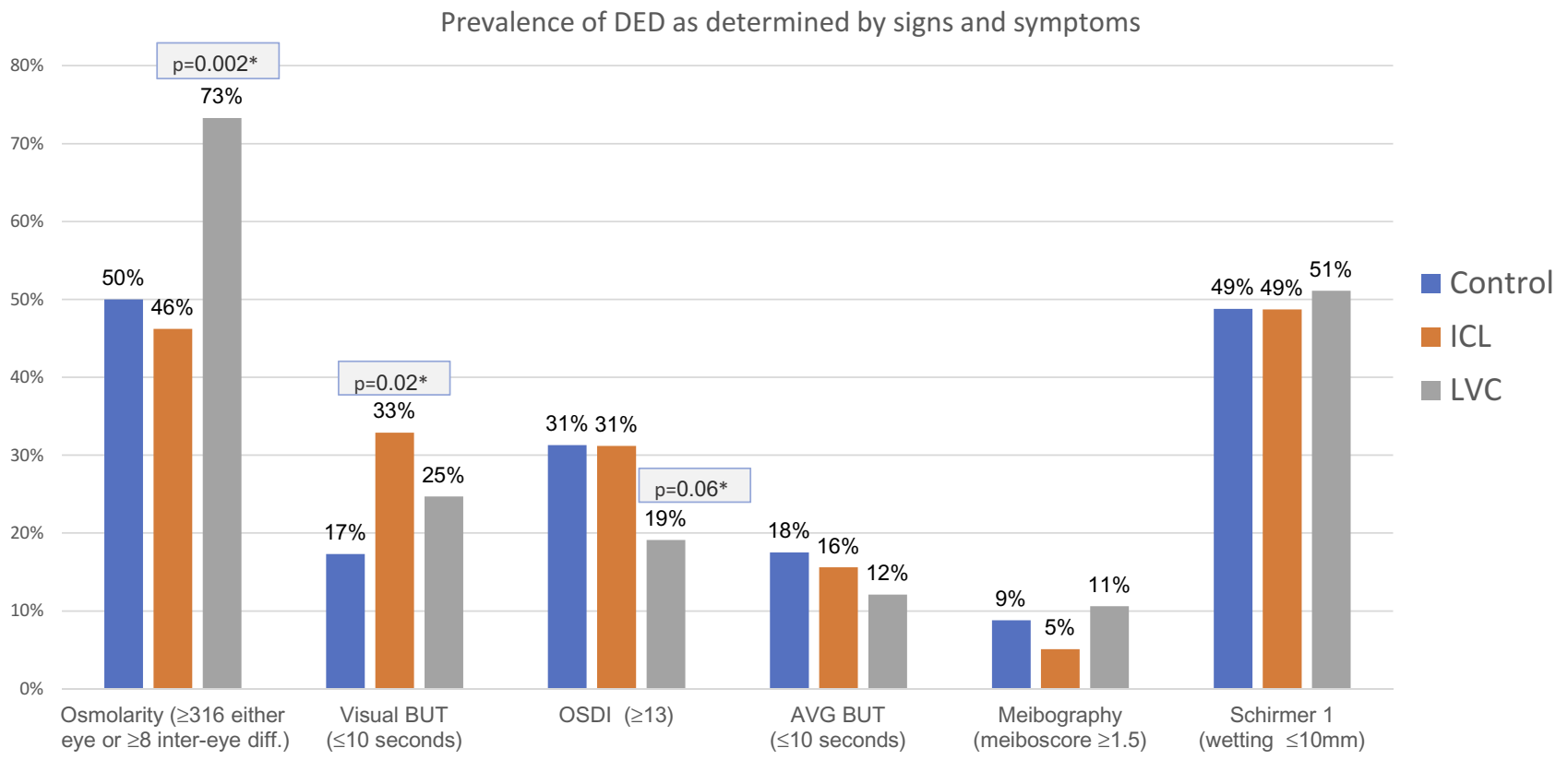

Figure I Comparing the prevalence of DED as determined by different tests between LVC or ICL and control group. Notes: *Pearson's $\chi^{2}$ : difference from control group.

Abbreviations: BUT, Break-up time; OSDI, ocular surface disease index; AVG, average; ICL, Implantable collamer lens; LVC, laser vision correction. 


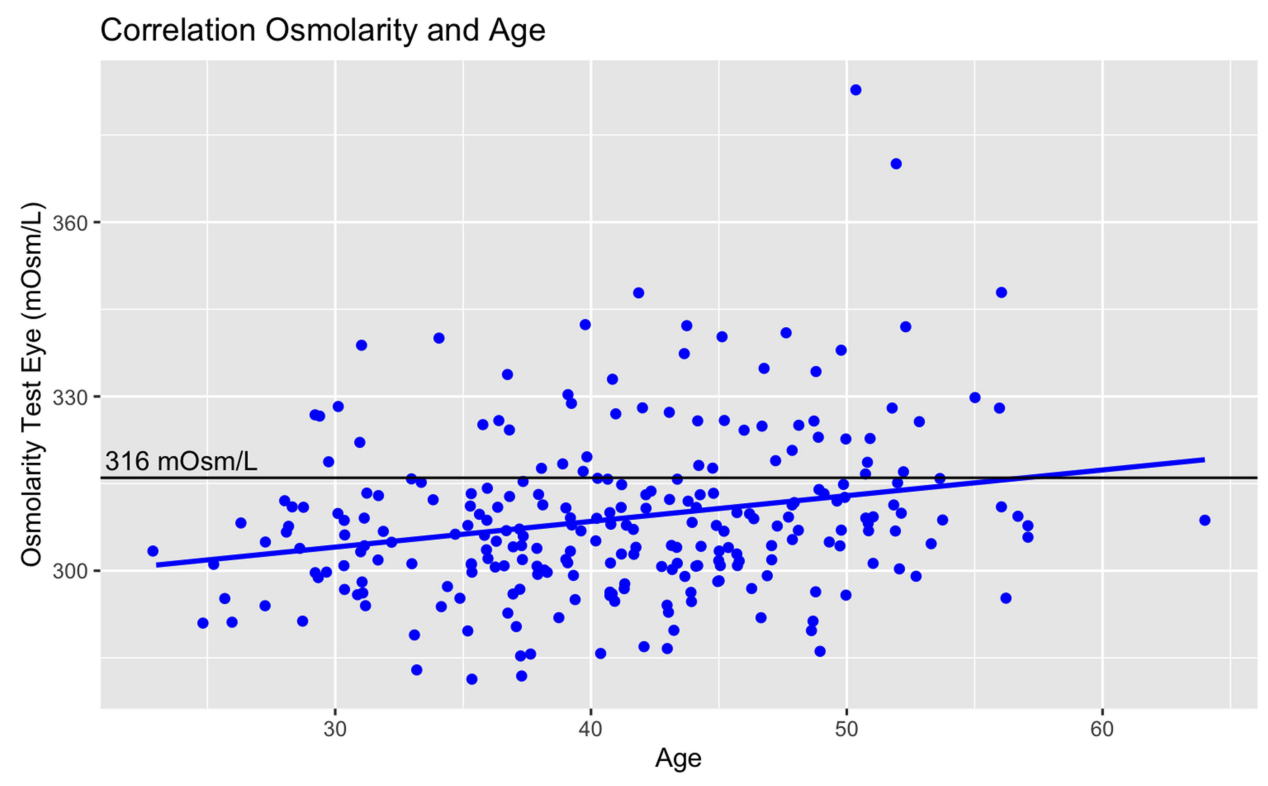

Figure 2 Example of weak correlations, here between osmolarity and age. Several outliers are influencing the fit. The single eye osmolarity cut-off value of $316 \mathrm{mOsm} / \mathrm{L}$ is shown for reference.

The mean of the dynamic OSI measures was significantly higher in the ICL group, but these measures were correlated to the minimum OSI (before tear film changes). The introduction of a pIOL into an optical system could reduce the optical quality of the system significantly, ${ }^{48}$ so the increased OSI values in the ICL group may be a result of reduced optical quality. The device software normalizes measurement to compensate for different levels of scatter, but this might not be sufficient in the case of a pIOL.

There was a tendency for fewer subjective symptoms in the LVC group. Studies have shown that subjective and objective symptoms may not agree due to differences in age, tolerance, environment and even long-standing dry eye which can reduce sensitivity. ${ }^{3,43}$ In post-LASIK patients, it may be that reduced symptoms are due to reduced sensitivity. A review report by Shtein found that studies of nerve morphology have shown reduced density 3-5 year after surgery. ${ }^{49}$ This strengthens the hypothesis that LASIK surgery can induce and even mask dry eye permanently due to incomplete nerve regeneration. In consequence, the recommendation in the DEWS II report on diagnosing DED by subjective symptoms and one homeostasis marker may not be optimal for post-LVC patients. ${ }^{7}$

We could not establish a significant correlation between osmolarity and other single dry eye tests. The lack of correlation between different diagnostic tests is likely a consequence of the multi-factorial nature of DED and the fact that different diagnostic tests reveal different aspects of the disease.$^{50,51}$ However, we did find that patients with two or more other indicators of DED showed a significant higher frequency of hyperosmolarity. Classification of dry eyes is usually based on several tests, but tear osmolarity has been shown to be the best single metric both to diagnose and classify DED and evidence indicates that tear hyperosmolarity contributes to, and is representative of, the mechanisms involved in the development and progression of DED. ${ }^{42,51} \mathrm{In}$ a review report by Potvin et al they found that a majority of the studies reviewed supported the use of tear osmolarity as a tool of diagnosis and severity grading. ${ }^{50}$

There are some limitations to the study. There was a risk of selection bias as patients were informed about the study on recruitment and patients with symptoms may have been more interested in participating, but the proportion of patients who agreed to participate was high and the subjective symptom score was low. Factors such as systemic or topical drugs and occupation should be the same across groups, but where not controlled and might have influenced our findings. There were significantly more females in the ICL group, though there was no correlation between sex and osmolarity. Also, there were significant differences in pre-MRSE, but only weak correlation between pre-MRSE and osmolarity. The study included patients with a large span of years since surgery and there were significant differences in this time span between groups, but there was no correlation between years since surgery and osmolarity. In addition, the first 
surgeries were as long as 15 years ago and surgical techniques may have changed, which might have influenced our findings relative to those that are more recent. Our study included only LASIK and Femto-LASIK surgeries, so we did not address whether LASEK or PRK affects dry eye.

\section{Conclusion}

Osmolarity differences suggested a significantly higher prevalence of DED in patients who underwent LVC 5 to 15 years ago than in a matched control group, though the LVC group had fewer subjective symptoms. The recommendation in the DEWS II report on diagnosing DED by subjective symptoms and one homeostasis marker may not be optimal for post-LVC patients. Hyperosmolarity increases the risk for tear film instability which is likely to be a confounding source of error for post-LVC IOL calculations. Further studies of post-LVC tear film quality are advocated. For instance, of interest is the potential effect of reduced tear quality on repeatability of measurements with different types of keratometers.

\section{Disclosure}

Bjørn Gjerdrum reports grants from The Research Council of Norway, grants from SkatteFUNN R\&D tax incentive scheme, grants from Memira AS, during the conduct of the study. The authors report no other conflicts of interest in this work.

\section{References}

1. Haigis W. Intraocular lens calculation after refractive surgery for myopia: haigis-L formula. $J$ Cataract Refract Surg. 2008;34 (10):1658-1663. doi:10.1016/j.jcrs.2008.06.029

2. Olsen T. Calculation of intraocular lens power: a review. Acta Ophthalmol Scand. 2007;85(5):472-485. doi:10.1111/j.1755-3768.2007.00879.x

3. Epitropoulos AT, Matossian C, Berdy GJ, Malhotra RP, Potvin R. Effect of tear osmolarity on repeatability of keratometry for cataract surgery planning. J Cataract Refract Surg. 2015;41(8):1672-1677. doi: $10.1016 /$ j.jcrs.2015.01.016

4. Craig JP, Nichols KK, Akpek EK, et al. TFOS DEWS II definition and classification report. Ocul Surf. 2017;15(3):276-283. doi:10.10 16/j.jtos.2017.05.008

5. Nelson JD, Craig JP, Akpek EK, et al. TFOS DEWS II introduction. Ocul Surf. 2017;15(3):269-275. doi:10.1016/j.jtos.2017.05.005

6. Bron AJ, de Paiva CS, Chauhan SK, et al. TFOS DEWS II pathophysiology report. Ocul Surf. 2017;15(3):438-510. doi:10.1016/j. jtos.2017.05.011

7. Wolffsohn JS, Arita R, Chalmers R, et al. TFOS DEWS II diagnostic methodology report. Ocul Surf. 2017;15(3):539-574. doi:10.1016/j. jtos.2017.05.001

8. Gomes JAP, Azar DT, Baudouin C, et al. TFOS DEWS II iatrogenic report. Ocul Surf. 2017;15(3):511-538. doi:10.1016/j.jtos.2017.05.004

9. Solomon KD, Fernandez de Castro LE, Sandoval HP, et al. LASIK world literature review: quality of life and patient satisfaction. Ophthalmology. 2009;116(4):691-701. doi:10.1016/j.ophtha.2008.12.037
10. Statista. Number of LASIK surgeries in the United States from 1996 to 2020. Statista; 2019. Available from: https://www.statista.com/statistics/271478/ number-of-lasik-surgeries-in-the-us/. Accessed December 08, 2019.

11. Solomon R, Donnenfeld ED, Perry HD. The effects of LASIK on the ocular surface. Ocul Surf. 2004;2(1):34-44. doi:10.1016/S15420124(12)70022-8

12. Ambrósio R, Tervo T, Wilson SE. LASIK-associated dry eye and neurotrophic epitheliopathy: pathophysiology and strategies for prevention and treatment. $J$ Refract Surg. 2008;24(4):396-407. doi:10.3928/1081597X-20080401-14

13. Xie W. Recent advances in laser in situ keratomileusis-associated dry eye. Clin Exp Optom. 2016;99(2):107-112. doi:10.1111/cxo.12361

14. Cohen E, Spierer O. Dry eye post-laser-assisted in situ keratomileusis: major review and latest updates. J Ophthalmol. 2018;2018:4903831.

15. Wilson SE, Ambrósio R. Laser in situ keratomileusis-induced neurotrophic epitheliopathy. Am $J$ Ophthalmol. 2001;132(3):405. doi:10.1016/S0002-9394(01)00995-3

16. Feng YF, Yu JG, Wang DD, et al. The effect of hinge location on corneal sensation and dry eye after LASIK: a systematic review and meta-analysis. Graefes Arch Clin Exp Ophthalmol. 2013;251(1):357-366.

17. Lee JB, Ryu CH, Kim J-H, Kim EK, Kim HB. Comparison of tear secretion and tear film instability after photorefractive keratectomy and laser in situ keratomileusis ${ }^{1}$. J Cataract Refract Surg. 2000;26 (9):1326-1331. doi:10.1016/S0886-3350(00)00566-6

18. Nettune GR, Pflugfelder SC. Post-LASIK tear dysfunction and dysesthesia. Ocul Surf. 2010;8(3):135-145. doi:10.1016/S15420124(12)70224-0

19. Shoja MR, Besharati MR. Dry eye after LASIK for myopia: incidence and risk factors. Eur J Ophthalmol. 2007;17(1):1-6. doi:10. 1177/112067210701700101

20. De Paiva CS, Chen Z, Koch DD, et al. The incidence and risk factors for developing dry eye after myopic LASIK. Am J Ophthalmol. 2006;141(3):438-445. doi:10.1016/j.ajo.2005.10.006

21. Dooley I, D'Arcy F, O'Keefe M. Comparison of dry-eye disease severity after laser in situ keratomileusis and laser-assisted subepithelial keratectomy. J Cataract Refract Surg. 2012;38(6):1058-1064. doi:10.1016/j.jcrs.2012.01.028

22. Wang X, Zhou X. Update on treating high myopia with implantable collamer lenses. Asia Pac J Ophthalmol (Phila). 2016;5(6):445-449. doi:10.1097/APO.0000000000000235

23. Dougherty PJ, Priver T. Refractive outcomes and safety of the implantable collamer lens in young low-to-moderate myopes. Clin Ophthalmol. 2017;11:273-277. doi:10.2147/OPTH

24. Naj AC, Liang G-L, Wu J, et al. Implantable collamer lens versus iris-fixed phakic intraocular lens implantation to correct myopia: a meta-analysis. PLoS One. 2014;9(8):e104649.

25. TearLab. Osmolarity System - User Manual. San Diego, CA: U.S.A. TearLab Corporation; 2017.

26. Gouvea L, Waring IGO, Brundrett A, Crouse M, Rocha KM. Objective assessment of optical quality in dry eye disease using a double-pass imaging system. Clin Ophthalmol. 2019;13:1991-1996. doi:10.2147/OPTH.S211584

27. Herbaut A, Liang H, Rabut G, et al. Impact of dry eye disease on vision quality: an optical quality analysis system study. Transl Vis Sci Technol. 2018;7(4):5. doi:10.1167/tvst.7.4.5

28. Monte's-Mico' R, Alio' JL, Charman WN. Postblink changes in the ocular modulation transfer function measured by a double-pass method. Invest Ophthalmol Vis Sci. 2005;46(12):4468-4473. doi:10.1167/iovs.05-0609

29. Schiffman RM, Christianson MD, Jacobsen G, Hirsch JD, Reis BL. Reliability and validity of the ocular surface disease index. Arch Ophthalmol. 2000;118(5):615-621. doi:10.1001/archopht.118.5.615

30. Oculus. Oculus Keratograph $5 M$ User Guide. Wetzlar, Germany: Oculus Optikergerâte; 2017.

31. Oculus. Oculus Keratograph 5M Instruction Manual. Wetzlar, Germany: Oculus Optikgeräte; 2017. 
32. Savini G. The challenge of dry eye diagnosis. Clin Ophthalmol. 2008;31.

33. Best N, Drury L, Wolffsohn JS. Clinical evaluation of the Oculus Keratograph. Cont Lens Anterior Eye. 2012;35(4):171-174. doi:10.10 16/j.clae.2012.04.002

34. Tian L, Qu JH, Zhang XY, Sun XG. Repeatability and reproducibility of noninvasive keratograph $5 \mathrm{M}$ measurements in patients with dry eye disease. $J$ Ophthalmol. 2016;2016:8013621.

35. Jiang Y, Ye H, Xu J, Lu Y. Noninvasive Keratograph assessment of tear film break-up time and location in patients with age-related cataracts and dry eye syndrome. J Int Med Res. 2014;42 (2):494-502. doi:10.1177/0300060513504701

36. Arita R, Itoh K, Maeda S, et al. Proposed diagnostic criteria for obstructive meibomian gland dysfunction. Ophthalmology. 2009;116 (11):2058-2063. doi:10.1016/j.ophtha.2009.04.037

37. Finis D, Ackermann P, Pischel N, et al. Evaluation of meibomian gland dysfunction and local distribution of meibomian gland atrophy by non-contact infrared meibography. Curr Eye Res. 2015;40 (10):982-989. doi:10.3109/02713683.2014.971929

38. de Monchy I, Gendron G, Miceli C, Pogorzalek N, Mariette X, Labetoulle M. Combination of the Schirmer I and phenol red thread tests as a rescue strategy for diagnosis of ocular dryness associated with sjögren's syndrome. Invest Ophthalmol Vis Sci. 2011;52 (8):5167-5173. doi:10.1167/iovs.10-6671

39. Hill WE, Wang L, Koch DD IOL power calculation in eyes that have undergone LASIK/PRK/RK, online calculator, version 4.9. Available from: http://iolcalc.ascrs.org/. Accessed December 10, 2019.

40. Vrijman V, Abulafia A, van der Linden JW, van der Meulen IJE, Mourits MP, Lapid-Gortzak R. Evaluation of different IOL calculation formulas of the ASCRS calculator in eyes after corneal refractive laser surgery for myopia with multifocal IOL implantation. $J$ Refract Surg. 2019;35(1):54-59. doi:10.3928/1081597X-20181119-01

41. Brenner LF, Gjerdrum B, Aakre BM, Lundmark PO, Nistad K. Presbyopic refractive lens exchange with trifocal intraocular lens implantation after corneal laser vision correction: refractive results and biometry analysis of 241 eyes. J Cataract Refract Surg. 2019;45:1404-1415. doi:10.1016/j.jcrs.2019.05.031
42. Lemp MA, Bron AJ, Baudouin C, et al. Tear osmolarity in the diagnosis and management of dry eye disease. Am J Ophthalmol. 2011;151(5):792-798. doi:10.1016/j.ajo.2010.10.032

43. Stapleton F, Alves M, Bunya VY, et al. TFOS DEWS II epidemiology report. Ocul Surf. 2017;15(3):334-365. doi:10.1016/j.jtos. 2017.05.003

44. Gupta PK, Drinkwater OJ, VanDusen KW, Brissette AR, Starr CE. Prevalence of ocular surface dysfunction in patients presenting for cataract surgery evaluation. J Cataract Refract Surg. 2018;44 (9):1090-1096. doi:10.1016/j.jcrs.2018.06.026

45. McGhee CN, Orr D, Kidd B, Stark C, Bryce IG, Anastas CN. Psychological aspects of excimer laser surgery for myopia: reasons for seeking treatment and patient satisfaction. $\mathrm{Br} J$ Ophthalmol. 1996;80(10):874. doi:10.1136/bjo.80.10.874

46. Toda I, Asano-Kato N, Hori-Komai Y, Tsubota K. Laser-assisted in situ keratomileusis for patients with dry eye. JAMA Ophthalmol. 2002;120(8):1024-1028.

47. Konomi K, Chen LL, Tarko RS, et al. Preoperative characteristics and a potential mechanism of chronic dry eye after LASIK. Invest Ophthalmol Vis Sci. 2008;49(1):168-174. doi:10.1167/iovs.07-0337

48. Artigas JM, Peris C, Felipe A, Menezo JL, Sanchez-Cortina I, LopezGil N. Modulation transfer function: rigid versus foldable phakic intraocular lenses. J Cataract Refract Surg. 2009;35(4):747-752. doi:10.1016/j.jers.2008.12.020

49. Shtein RM. Post-LASIK dry eye. Expert Rev Ophthalmol. 2011;6 (5):575-582. doi: $10.1586 /$ eop. 11.56

50. Potvin R, Makari S, Rapuano C. Tear film osmolarity and dry eye disease: a review of the literature. Clin Ophthalmol. 2015;2039. doi:10.2147/OPTH

51. Sullivan BD, Crews LA, Messmer EM, et al. Correlations between commonly used objective signs and symptoms for the diagnosis of dry eye disease: clinical implications. Acta Ophthalmol. 2014;92 (2):161-166. doi:10.1111/aos.12012
Clinical Ophthalmology

\section{Publish your work in this journal}

Clinical Ophthalmology is an international, peer-reviewed journal covering all subspecialties within ophthalmology. Key topics include: Optometry; Visual science; Pharmacology and drug therapy in eye diseases; Basic Sciences; Primary and Secondary eye care; Patient Safety and Quality of Care Improvements. This journal is indexed on PubMed

\section{Dovepress}

Central and CAS, and is the official journal of The Society of Clinical Ophthalmology (SCO). The manuscript management system is completely online and includes a very quick and fair peer-review system, which is all easy to use. Visit http://www.dovepress.com/ testimonials.php to read real quotes from published authors. 